\title{
Synchronous Hepatocellular Carcinoma and Cholangiocarcinoma Arising in Two Different Dysplastic Nodules
}

\author{
Youngmee Kwon, M.D., Seung Kyu Lee, M.D., Jung-Sun Kim, M.D., Jae Y. Ro, M.D., Eunsil Yu, M.D. \\ Departments of Pathology and Surgery, University of Ulsan College of Medicine, Asan Medical Center, \\ Seoul, Korea
}

We present the first reported case of explant cirrhotic liver that had synchronous cholangiocarcinoma and hepatocellular carcinoma arising in two different high-grade dysplastic nodules. The patient was a 55-year-old woman who had hepatitis B virus-associated liver cirrhosis for 3 years. The moderately differentiated cholangiocarcinoma occurred in high-grade dysplastic nodule with a 1.7-fold cell density compared with that of cirrhotic nodule. The hepatocellular carcinoma arose in a nodule-innodule pattern within a peripherally low-grade and centrally high-grade dysplastic nodule and had a 2.7-fold cell density compared with that of cirrhotic nodule. By immunohistochemistry, the tumor cells of the cholangiocarcinoma as well as bile ductular cells in dysplastic nodule were diffusely positive for cytokeratin 7 , whereas hepatocellular carcinoma cells and dysplastic hepatocytes were negative for cytokeratin 7 . The c-kit-positive hepatic progenitor cells were singly scattered between hepatocytes, and their number was highest in cirrhotic nodule and decreased in dysplastic nodule, whereas they were absent in cholangiocarcinoma and hepatocellular carcinoma arising in dysplastic nodules. Proliferation indices were progressively increased in cirrhotic nodule, dysplastic nodule, and cholangiocarcinoma or hepatocellular carcinoma, sequentially. These observations indicate that cholangiocarcinoma as well as hepatocellular carcinoma can develop in dysplastic nodule and that hepatic progenitor cells might play a role in the early stage of cholangiocarcinogenesis and hepatocarcinogenesis.

Copyright (C) 2002 by The United States and Canadian Academy of Pathology, Inc.

VOL. 15, NO. 10, P. 1096, 2002 Printed in the U.S.A.

Date of acceptance: June 7, 2002.

Address reprint requests to: Eunsil Yu, M.D., Department of Pathology, Asan Medical Center, University of Ulsan College of Medicine, 388-1 Pungnapdong Songpa-gu, Seoul 138-736, Korea; e-mail: esyu@www.amc.seoul.kr; fax: 02-472-7898.

DOI: 10.1097/01.MP.0000027622.74779.AE
KEY WORDS: Cholangiocarcinoma, Dysplastic nodule, Hepatic progenitor cells, Hepatocellular carcinoma.

Mod Pathol 2002;15(10):1096-1101

High-grade dysplastic nodule is known as a preneoplastic lesion of hepatocellular carcinoma (1-3). Small hepatocellular carcinomas frequently occur within high-grade DN, displaying nodule-in-nodule pattern $(2,4)$ that supports multistep hepatocarcinogenesis. Cholangiocarcinoma occurs in intrahepatic bile duct epithelial cells, and some cholangiocarcinomas are confined within intrahepatic bile ducts (5). Although carcinogenesis of intrahepatic cholangiocarcinoma and hepatocellular carcinoma has not been elucidated in human, oval cells are known to be a counterpart of human hepatic progenitor cells and to be closely associated with liver damage and carcinogenesis in animal experiments (6-8). We present the first case of small cholangiocarcinoma arising in high-grade dysplastic nodule in addition to nodule-in-nodule-type hepatocellular carcinoma arising in high-grade dysplastic nodule, and this case suggests that human hepatic progenitor cells can play a role in the early stage of cholangiocarcinogenesis and hepatocarcinogenesis.

\section{CASE REPORT}

A 55-year-old woman visited a local clinic in 1999 with a complaint of epigastric pain. There, she was diagnosed as having hepatitis B virus-associated liver cirrhosis. Thus, she was referred to the Asan Medical Center, Seoul, Korea for management of liver cirrhosis. Abnormal initial laboratory data were as follows: AST/ALT 87/84 IU/L, alkaline phosphatase/r-GT 218/59 IU/L, and $\alpha$-fetoprotein, $101 \mathrm{ng} / \mathrm{mL}$. HBsAg and HBeAg were found in the serum, but HBsAb was absent. No other abnormalities were found with regard to peripheral blood, blood chemistry, and urine. Ultrasonography and computed tomography (CT) showed liver cirrhosis 
with ascites and splenomegaly. Nine months later, a few small enhancing nodules were detected in the hepatic dome and the right posterior inferior segment (S6) on a repeated CT scan, which suggested a hepatocellular carcinoma. Five months later, follow-up CT scan exhibited an arterial enhancing mass of $4.5 \mathrm{~cm}$ in the greatest dimension in S6, two additional small enhancing nodules in the right anterior superior segment (S8; Figs. 1A, 2A) and the fourth enhancing nodule in the left lateral inferior segment (S3). Transarterial chemoembolization was done for the largest mass in S6. One month later, liver transplantation was performed.

\section{MATERIALS AND METHODS}

The explant liver specimen was formalin fixed, paraffin embedded, cut into $4-\mu \mathrm{m}$ sections, and stained with hematoxylin and eosin. Histochemical stainings including mucicarmine and Alcian blue stainings at $\mathrm{pH} 2.5$ were performed to detect intracytoplasmic or extracellular mucin production. For immunohistochemistry, formalin-fixed, paraffinembedded tissue sections were cut at $4 \mu \mathrm{m}$, heated at $60^{\circ} \mathrm{C}$, deparaffinized in xylene, and hydrated in a graded series of alcohols. When necessary, antigen retrieval was performed using microwaving in citrate buffer for 10 minutes. Endogenous peroxidase activity was inactivated by incubation in $3 \% \mathrm{H}_{2} \mathrm{O}_{2}$ for 10 minutes. Nonspecific binding sites were blocked by incubating in $10 \%$ normal goat serum diluted with phosphate-buffered saline. Tissue sections were then incubated with the primary antibodies against cytokeratin 7 (CK 7; 1:200, DAKO, Glostrup, Denmark), AE1/AE3 (1: 200, Zymed, San Francisco, CA), carcinoembryonic antigen (CEA; 1:200, DAKO), Ki-67 (1:200, DAKO), CD34 (1:2,500, Immunotech, Marseille, France), and c-kit (1:400, DAKO) for 60 minutes at room temperature. All sections were sequentially treated with biotinylated anti-rabbit or anti-mouse immunoglobulin for 10 minutes, peroxidase-labeled streptavidin for $10 \mathrm{~min}$ utes, and diaminobenzidine in the presence of hydrogen peroxide. Sections were counterstained with light hematoxylin (Gill 2; Richard Allen Scientific, Kalamazoo, MI). A negative control was included in which the primary antibodies were replaced by nonhuman reactive mouse immunoglobulin $\mathrm{G}$.

\section{RESULTS}

The explant liver was atrophic and cirrhotic, measuring $22 \times 11 \times 6 \mathrm{~cm}$ and weighing $750 \mathrm{~g}$. In S8, two masses, measuring $1.7 \mathrm{~cm}$ (Tumor 1, Fig. 1B) and $2.0 \mathrm{~cm}$ (Tumor 2, Fig. 2B) in the greatest dimension, were founded. The two nodules were $2.0 \mathrm{~cm}$ away from each other. Cut surfaces of both masses were relatively well demarcated and bulging out from the adjacent cirrhotic nodules (Figs. 1B-1, 2B-1). Tumor 1 showed a nodule-in-nodule pattern and had a round pinkish yellow central nodule (Fig. 1B-4) in a background of greenish tan nodule (Fig. 1B-2) and intervening light brown, crescent-shaped thin rim (Fig. 1B-3). Tumor 2 was brownish at the periphery (Fig. 2B-2) and had an ill-defined lobulated yellowish white nodule at the center (Fig. 2B$3)$. The chemoembolized mass in $S 6(4.5 \mathrm{~cm}$ at greatest dimension) was greenish yellow and totally necrotic. The fourth nodule in $\mathrm{S} 3(1.0 \mathrm{~cm})$ was homogeneously yellowish tan and focally hemorrhagic. Portal veins, hepatic arteries, and bile ducts were unremarkable, with no evidence of tumor.

Microscopically, Tumor 1 showed three different histologic features as a nodule-in-nodule pattern. The peripheral portion was of low-grade dysplastic nodule with mild fatty change (Fig. 1C-2) and increased cell density, approximately 1.2 -fold of that in surrounding cirrhotic nodules (Fig. 1C-1; Table 1). There were foci of small ductular proliferation that were closely associated with leukocytic infiltration and degenerative changes of adjacent hepatocytes. The mid portion was of high-grade dysplastic nodule with significantly increased cell density, approximately 2.2 -fold of that in cirrhotic nodules, cytoplasmic basophilia, a high nuclear-cytoplasmic ratio, and nuclear hyperchromasia (Fig. 1C-3). However, neither acini nor trabeculae were developed. The central nodule was highly cellular (2.7fold of that in cirrhotic nodule) and consisted of microtrabeculae of well differentiated hepatocellular carcinoma (Fig. 1C-4).

The cell density of the peripheral nodule of Tumor 2 was 1.7 -fold of that in cirrhotic nodules, which was compatible with that of high-grade dysplastic nodule (Fig. 2C-2, Table 1). The central portion revealed cholangiocarcinoma with glandular or ductular structures and rare intracytoplasmic, but confluent intraluminal mucin, and stromal fibrosis. Alcian blue staining at $\mathrm{pH} 2.5$ highlighted intracellular and luminal mucin (Fig. 2C-3, C-4). No viable tumor cells were found in the chemoembolized mass in S6. The nodule in S3 was hepatocellular carcinoma, Edmondson-Steiner Grade 2, entirely consisting of well-developed trabeculae and sinusodial structures.

By immunohistochemical stainings, the hepatocellular carcinoma and cholangiocarcinoma were differently stained for CK 7 and CEA. The hepatocellular carcinoma was negative for both CK 7 (Fig. 1D-1) and CEA. In the surrounding low-grade dysplastic nodule, there were scattered CK-7-positive isolated cells and ductular structures (Fig. 1D-2). The cholangiocarcinoma was diffusely but variably positive for CK 7, with peripheral portion of the tumor being much more strongly stained (Fig. 

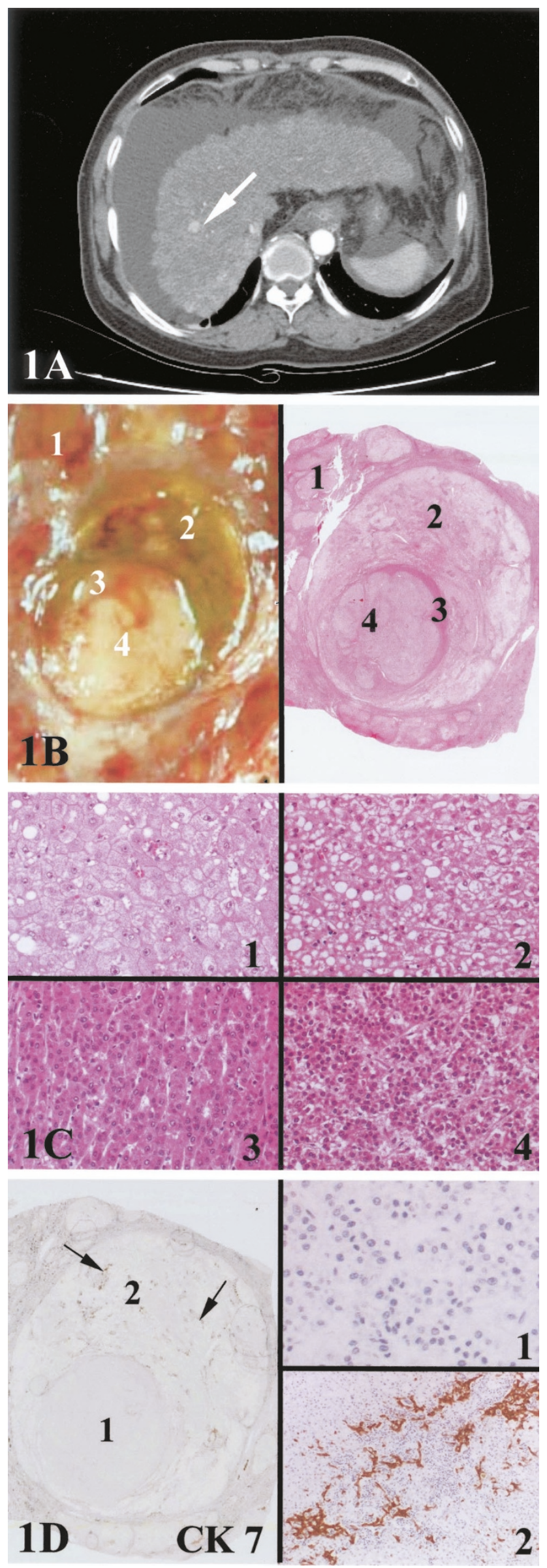

2D-1, D-2). Immunoreactivity for CEA was strongly positive in the cholangiocarcinoma but was negative in hepatocellular carcinoma. The c-kit-positive cells excluding endothelial cells were easily found in the fibrous septa around cirrhotic nodules and portal areas within low-grade dysplastic nodule. The number of c-kit-positive cells was highest in cirrhotic nodules and was progressively decreased in low- and high-grade dysplastic nodules (Fig. 2D, Table 1). In cholangiocarcinoma and hepatocellular carcinoma, c-kit-positive cells were absent.

Proliferation indices were significantly higher in both hepatocellular carcinoma and cholangiocarcinoma than were those of dysplastic nodule and cirrhotic nodule. However, there were no differences between hepatocellular carcinoma and cholangiocarcinoma (Table 1).

\section{DISCUSSION}

This case represents the first reported case of synchronous small hepatocellular carcinoma and small cholangiocarcinoma arising in the different high-grade dysplastic nodules. The hepatocellular carcinoma arising in dysplastic nodule is considered to develop independently from the totally necrotic hepatocellular carcinoma and the other small hepatocellular carcinoma because it reveals a nodulein-nodule growth pattern and gradual increase of cell density and atypia, compared with the background low- and high-grade dysplastic nodules.

Although small hepatocellular carcinomas occur in dysplastic nodule as a nodule-in-nodule type (2, 4), small cholangiocarcinoma arising in a dysplastic nodule has not been reported yet. Only one case of combined hepatocellular carcinoma and cholangiocarcinoma in atypical adenomatous hyperplasia was reported; however, histopathologic features of atypical adenomatous hyperplasia were not presented in detail (9).

In the present case, a total of three dysplastic nodules were present; one low-grade dysplastic nodule and two high-grade dysplastic nodules.

FIGURE 1. Hepatocellular carcinoma in dysplastic nodule. A, computed tomography of the liver discloses an enhancing nodule in S8. B, cut surface and 1:1 microscopy of a dysplastic nodule with hepatocellular carcinoma. Hepatocellular carcinoma occupies almost the entire nodule, and crescent-shaped high-grade dysplastic nodule is shown at the periphery. C, light-microscopic features of hepatocellular carcinoma and dysplastic nodules. Note progressive increase in cell density and cellular atypia as well as structural abnormalities in hepatocellular carcinoma (4) compared with cirrhotic nodule (1), lowgrade dysplastic nodule (2), and high-grade dysplastic nodule (3). D, immunohistochemical analysis for cytokeratin 7 . Hepatocellular carcinoma is totally negative for cytokeratin 7 (1), and there are aggregates of cytokeratin-positive cells forming poorly developed ductules in low-grade dysplastic nodule (2, arrows). 

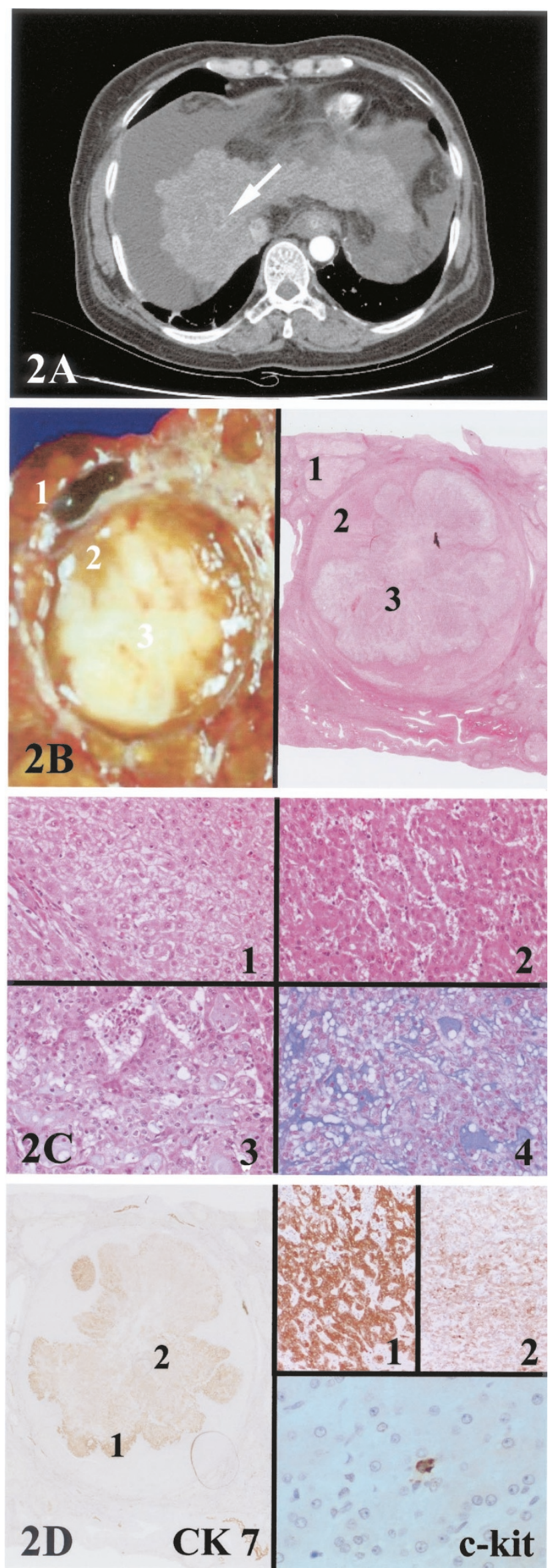

Small cholangiocarcinoma and hepatocellular carcinoma occurred in two different high-grade dysplastic nodules. One of the high-grade dysplastic nodules was associated with a low-grade dysplastic nodule and hepatocellular carcinoma (Tumor 1). High-grade dysplastic nodule has been regarded as a precancerous lesion of hepatocellular carcinoma; however, the actual developmental mechanism of hepatocellular carcinoma from dysplastic nodule as a nodule-in-nodule type has not been firmly established $(10,11)$. Theise et al. have suggested dysplastic nodule development as a clonal expansion in advance or parallel to cirrhosis $(12,13)$, and the dysregulation of apoptosis or proliferation might play an important role in the process of hepatocarcinogenesis (14).

Among many criteria for differential diagnosis of dysplastic nodule and well-differentiated hepatocellular carcinoma, cell density is considered one of the objective criteria (10). Cell density is determined by the difference between cell proliferation and death. Thus, increased cell density in preneoplastic or neoplastic lesions is attributable to either increased proliferation or decreased apoptosis. In the present case, the marked increase of Ki-67labeling index supported the significant increase of cell density in high-grade dysplastic nodule as well as hepatocellular carcinoma and cholangiocarcinoma. Cell density of high-grade dysplastic nodule with hepatocellular carcinoma was much higher than the suggested criteria (10); however, structural atypia such as acinar or trabecular arrangement was absent. Cell density in the high-grade dysplastic nodule with cholangiocarcinoma ranged between that of low-grade dysplastic nodule and high-grade dysplastic nodule with hepatocellular carcinoma, but thickened cell cords and occasional acinar structure were helpful for grading this dysplastic nodule as a high-grade dysplastic nodule.

Human hepatic progenitor cells are similar to oval cells of the rodent liver in location, morphological and immunohistochemical phenotypes, and functional aspects (15-20). Human hepatic progenitor cells have been identified in several

FIGURE 2. Cholangiocarcinoma in dysplastic nodule. A, computed tomography of the liver shows a nonenhancing nodule with peripheral enhancement in S8. B, cut surface and 1:1 microscopy of a dysplastic nodule with cholangiocarcinoma. The yellowish white and firm cholangiocarcinoma is located in the background of high-grade dysplastic nodule. C, light-microscopic features of cholangiocarcinoma and dysplastic nodule. Note progressive increase in cell density and cellular atypia as well as structural atypia in cholangiocarcinoma (3), compared with cirrhotic nodule (1) and high-grade dysplastic nodule (2). Cholangiocarcinoma displays glands with luminal and stromal mucin (4; Alcian blue staining). D, immunohistochemical analysis for cytokeratin 7 and c-kit. Cholangiocarcinoma is diffusely positive or cytokeratin 7 , but immunoreactivity was much stronger at the periphery (1), compared with that at the center of the tumor (2). A few c-kit-positive progenitor cells are scattered in dysplastic nodule. 
TABLE 1. Cell Density, Proliferation Index, and c-kit-positive Hepatic Progenitor Cells in Cirrhotic Nodule, Dysplastic Nodule, Hepatocellular Carcinoma, and Cholangiocarcinoma

\begin{tabular}{|c|c|c|c|}
\hline Diagnosis & Cell Density & $\begin{array}{l}\text { Proliferation } \\
\text { Index }\end{array}$ & $\begin{array}{c}\text { Hepatic } \\
\text { Progenitor Cells }\end{array}$ \\
\hline Cirrhotic nodule & $70 \pm 13$ & 16 & 7 \\
\hline Low-grade dysplastic nodule & $86 \pm 24$ & 15 & 4 \\
\hline High-grade dysplastic nodule with hepatocellular carcinoma & $156 \pm 18$ & 39 & 2 \\
\hline Hepatocellular carcinoma & $193 \pm 17$ & 104 & 0 \\
\hline High-grade dysplastic nodule with cholangiocarcinoma & $120 \pm 8$ & 30 & 2 \\
\hline Cholangiocarcinoma & $197 \pm 37$ & 107 & 0 \\
\hline
\end{tabular}

Cell density, average number of cells in one capture field of $\times 20$ objective lens; proliferation index, number of Ki-67-positive cells per 1000 cells; hepatic progenitor cells, number of c-kit-positive cells per 1000 cells.

human liver diseases including chronic viral hepatitis, hepatitis B virus-induced cirrhosis, focal nodular hyperplasia, small cell dysplastic foci, and hepatocellular adenoma (21-24). The presence of hepatic progenitor cells in preneoplastic lesions of hepatocellular carcinoma and overlapping morphology and immunohistochemical phenotype of poorly and moderately differentiated hepatocellular carcinoma with hepatic progenitor cells $(25,26)$ indicate that hepatic progenitor cells may play an important role in the development of human liver cell tumor. Among various markers of hepatic progenitor cells such as CK 7, AK19, OV-6, chromogranin A, and c-kit, only c-kit was applied, and c-kit-positive cells were counted as hepatic progenitor cells in the present case. Anastomosing cords or ductules, lined by flattened cells with scanty cytoplasm in the low-grade dysplastic nodule with hepatocellular carcinoma, were positive for CK 7 but negative for c-kit. Thus, they are considered either reactive bile ductules or intermediate hepatocyte-like cells that were defined by Libbrecht et al. (24). The average number of c-kit-positive cells per 1000 hepatocytes was significantly decreased in high-grade dysplastic nodule, and c-kit-positive cells were completely absent in cholangiocarcinoma and hepatocellular carcinoma. This inverse correlation of the number of hepatic progenitor cells with the multistep tumorigenesis of cholangiocarcinoma and hepatocellular carcinoma supports the hypothesis that hepatic progenitor cells can play a role in carcinogenesis of the liver and suggests a possible mechanism in that preexisting stem cells might differentiate to either hepatocellular carcinoma or cholangiocarcinoma.

\section{REFERENCES}

1. Arakawa M, Kage M, Sugihara S, et al. Emergence of malignant lesions within an adenomatous hyperplastic nodule in cirrhotic liver: observation in five cases. Gastroenterology 1986;91:198-208.
2. Takayama T, Makuuchi M, Hirohashi S. Malignant transformation of adenomatous hyperplasia to hepatocellular carcinoma. Lancet 1990;336:1150-3.

3. Kaji K, Terada T, Nakanuma Y. Frequent occurrence of hepatocellular carcinoma in cirrhotic livers after surgical resection of atypical adenomatous hyperplasia (borderline hepatocellular lesion): a follow-up study. Am J Gastroenterol 1994;89:903-8.

4. Sakamoto M, Hirohashi S, Shimosato Y. Early stages of multistep hepatocarcinogenesis: adenomatous hyperplasia and early hepatocellular carcinoma. Hum Pathol 1991; 22:172-8.

5. Ishak KG, Goodman ZD, Stocker JT. Intrahepatic cholangiocarcinoma and other malignant biliary tumors. In: Rosai J, editor. Atlas of tumor pathology. Tumors of the liver and intrahepatic bile ducts. 3rd series, Fascicle 38. Washington, D.C.: Armed Forces of Institute of Pathology; 2001. p. 245-70.

6. Pack R, Heck R, Dienes HP, et al. Isolation, biochemical characterization, long-term culture, and phenotype modulation of oval cells from carcinogen-fed rats. Exp Cell Res 1993;204:198-209.

7. Sell S. Liver stem cells. Mod Pathol 1994;7:105-12.

8. Paku S, Schnur J, Nagy P, et al. Origin and structural evolution of the early proliferating oval cells in rat liver. Am J Pathol 2001;158:1313-23.

9. Harada K, Terada T, Nakanuma Y, et al. A case of small combined hepatocellular and cholangiocellular carcinoma arising in a nodule of atypical adenomatous hyperplasia of the liver. Am J Gastroenterol 1993;88:1968-9.

10. International Working Party. Terminology of nodular hepatocellular lesions. Hepatology 1995;22:983-93.

11. Hytiroglou P, Theise ND, Schwartz M, et al. Macroregenerative nodules in a series of adult cirrhotic liver explants: issues of classification and nomenclature. Hepatology 1995;21:703-8.

12. Theise ND, Lapook JD, Thung SN. A macroregenerative nodule containing multiple foci of hepatocellular carcinoma in a noncirrhotic liver. Hepatology 1993;17:993-6.

13. Theise N. Macroregenerative (dysplastic) nodules and hepatocarcinogenesis: theoretical and clinical considerations. Semin Liver Dis 1995;15:360-71.

14. Park YN, Chae KJ, Kim YB, et al. Apoptosis and proliferation in hepatocarcinogenesis related to cirrhosis. Cancer 2001;92: 2733-8.

15. Roskams T, De Vos R, Van Eyken P, et al. Hepatic OV-6 expression in human liver disease and rat experiments: evidence for hepatic progenitor cells in man. J Hepatol 1998;29:455-63.

16. Libbrecht L, Desmet V, Van Damme B, et al. Deep intralobular extension of human hepatic progenitor cells correlates with parenchymal inflammation in chronic viral hepatitis: can progenitor cells migrate? J Pathol 2000; 192:373-8. 
17. Vessey CJ, de la Hall PM. Hepatic stem cells: a review. Pathology 2001;33:130-41.

18. Croby HA, Kelly DA, Strain AJ. Human hepatic stem-like cells isolated using c-kit or CD34 can differentiate into biliary epithelium. Gastroenterology 2001;120:534-44.

19. Mitaka T. Hepatic stem cells: from bone marrow cells to hepatocytes. Biochem Biophys Res Commun 2001;281: $1-5$.

20. Theise ND, Mimmakayalu M, Gardner R, et al. Liver from bone marrow in humans. Hepatology 2000;32:11-6.

21. Hsia CC, Evarts RP, Nakatsukasa $\mathrm{H}$, et al. Occurrence of oval-type cells in hepatitis B virus-associated human hepatocarcinogenesis. Hepatology 1992;16:1327-33.

22. Libbrecht L, Desmet V, Van Damme B, et al. The immunohistochemical phenotype of dysplastic foci in human liver: correlation with putative progenitor cells. J Hepatol 2000;33: 76-84.

23. Roskams T, De Vos R, Desmet V. "Undifferentiated progenitor cells" in focal nodular hyperplasia of the liver. Histopathology 1996;28:291-9.

24. Libbrecht L, De Vos R, Cassiman D, et al. Hepatic progenitor cells in hepatocellular adenomas. Am J Surg Pathol 2001;25: 1388-96.

25. Wu PC, Lai VC, Fang JW, et al. Hepatocellular carcinoma expressing both hepatocellular and biliary markers also express cytokeratin 14, a marker of bipotential progenitor cells. J Hepatol 1999;31:965-6.

26. Boppana S, Miller WM, Park YN, et al. Cytokeratin 19 expression in hepatocellular carcinoma [abstract]. Mod Pathol 2000;13:160.

\section{Erratum}

In the September issue of Modern Pathology, several articles had the wrong copyright year. The correct reference information is as follows:

Hasebe T, Sasaki S, Imoto S, Ochiai A. Characteristics of tumors in lymph vessels play an important role in the tumor progression of invasive ductal carcinoma of the breast: a prospective study. Mod Pathol 2002;15(9):904-913.

Cioc AM, Nuovo GJ. Histologic and in situ viral findings in the myocardium in cases of sudden, unexpected death. Mod Pathol 2002;15(9):914-922.

Mikami Y, Hata S, Kiyokawa T, Manabe T. Expression of CD10 in malignant Müllerian mixed tumors and adenosarcomas: an immunohistochemical study. Mod Pathol 2002;15(9):923-930.

Mayr D, Kaltz-Wittmer C, Arbogast S, Amann G, Aust DE, Diebold J. Characteristic pattern of genetic aberrations in ovarian granulosa cell tumors. Mod Pathol 2002;15(9):951-957.

Macabeo-Ong M, Ginzinger DG, Dekker N, McMillan A, Regezi JA, Wong DTW, Jordan RCK. Effect of duration of fixation on quantitative reverse transcription polymerase chain reaction analyses. Mod Pathol 2002;15(9):979-987.

Nagao T, Serizawa H, Iwaya K, Shimizu T, Sugano I, Ishida Y, Yamazaki K, Shimizu M, Itoh T, Konno A, Ebihara Y. Keratocystoma of the parotid gland: a report of two cases of an unusual pathologic entity. Mod Pathol 2002;15(9):1005-1010. 